First operation of ECR i on source at Kochi Uni versity of Technol ogy

\begin{tabular}{|l|l|}
\hline 著者 & $\begin{array}{l}\text { Monot a Sadao, Noj i r i Yoi chi , Sai har a M wako, } \\
\text { Sakanmt o Asako, Hanagawa Hi sayoshi, Hanaguchi } \\
\text { Kensuke }\end{array}$ \\
\hline $\begin{array}{l}\text { j our nal or } \\
\text { publ i cat i on t i tl e }\end{array}$ & Revi ew of Sci ent i f i c I nst r ument s \\
\hline vol une & 75 \\
\hline nunber & 5 \\
\hline page r ange & $1497-1498$ \\
\hline year & $2004-05-13$ \\
\hline URL & ht t p: //hdl . handl e. net /10173/611 \\
\hline
\end{tabular}




\title{
First operation of ECR ion source at Kochi University of Technology
}

\author{
Sadao Momota, ${ }^{\text {a) }}$ Yoichi Nojiri, Miwako Saihara, Asako Sakamoto, Hisayoshi Hamagawa, \\ and Kensuke Hamaguchi \\ Kochi University of Technology, Miyano-kuchi, Tosayamada-cho, Kochi, 782-8502, Japan
}

(Presented on 8 September 2003; published 13 May 2004)

\begin{abstract}
To study nano-scale manufacturing using highly charged ion beams, a facility to produce and irradiate heavy ion beams has been installed at Kochi University of Technology (KUT). The facility includes an ECR ion source (ECRIS), a beam transport and analysis system, and an irradiation system. The first beam was extracted from ECRIS in January 2003. To evaluate the performance of ECRIS, the measurements of the current and mass spectrum of ion beams as a function of the voltage for the beam extraction and of the rf power have been carried out. It is concluded from the present results that the combined use of the ECRIS and acceleration system with the transport and analysis system build at KUT works normally. (C) 2004 American Institute of Physics.
\end{abstract}

[DOI: $10.1063 / 1.1690450]$

\section{INTRODUCTION}

Recently, heavy ion beams, which were mainly applied to basic science such as atomic physics and nuclear physics, have been applied to other various fields. In particular, the formation of nanostructures is one field to which heavy ion beams are energetically applied. For example, the ion-beam based lithography has been applied to the fabrication of electric materials and devices. ${ }^{1,2}$ In almost all of those applications, only single charged ions were utilized. Highly charged ions have very high activity caused by their large reaction cross sections and large Coulomb potential. The high activity of highly charged ions causes a dramatic change in the surface of an irradiated material, which was studied in atomic physics. ${ }^{3}$ It is implied that the application of highly charged ions leads to more unique and efficient fabrication of nanostructures. To develop a technique for the formation of nanostructures by using highly charged ions, a facility to produce and irradiate highly charged ion beams was built at Kochi University of Technology (KUT). For this purpose, ease of changing the ion species and high productivity of highly charged ions are indispensable. An ECR ion source (ECRIS) fulfills the above-noted requirements.

\section{EXPERIMENTAL SETUP}

The facility includes an ECRIS and acceleration system. a transport and analysis system, and an irradiation system. A $10 \mathrm{GHz}$ NANOGAN, which is an ECRIS developed by PANTECHNIK Co. ${ }^{4.5}$ was installed. The rf power $\left(P_{\mathrm{rf}}\right)$ applied to NANOGAN is up to $80 \mathrm{~W}$. An extraction voltage $\left(V_{E}\right)$ of up to $30 \mathrm{kV}$ is applied, and an acceleration voltage $\left(V_{A}\right)$ of up to $100 \mathrm{kV}$ is applied to the HV platform. For easy handling and a compact install, an ECRIS and acceleration system are installed in a short length $(L \sim 1 \mathrm{~m})$. The transport and analysis system includes an einzel lens, an electric $Q$ lens, and a dipole magnet to perform a mass analysis. Two Faraday cups are placed so as to measure the beam current

${ }^{a)}$ Electronic mail: momota.sadao@kochi-tech.ac.jp both before and after the dipole magnet. The analyzed beams will be led to a radiation system, which is now being developed. The first beam was extracted from NANOGAN in January 2003. To check the performance of the facility, the current and the mass spectrum of ion beams extracted from NANOGAN were observed as a function of $V_{E}$ and $P_{\text {rf }}$.

\section{MEASUREMENTS}

To check the beam extraction, the total current of the ion beam extracted from NANOGAN $I_{\text {total }}$, into which Ar gas was fed, was observed as a function of $V_{E}$ and $P_{\text {rf }}$ with a Faraday cup placed before the dipole magnet. The typical results are shown in Figs. 1 and 2. The measured $I_{\text {total }}$ increases with $V_{E}$ and then saturates as can be seen in Fig. 1. The increasing part can be well reproduced by a power function, $I_{\text {total }}=A V_{E}^{B}$, with a solid line. The index of a power function derived from fitting $B=1.61$ (10) is consistent with that derived from the Chaild-Langmuir equation. In Fig. 2, it is found that the increasing part does not depend on $P_{\mathrm{rf}}$ and that the saturation value of $I_{\text {total }}\left(I_{\text {sat }}\right)$ increases with $P_{\mathrm{rf}}$. A correlation between $I_{\text {sat }}$ and $P_{\text {rf }}$ can be clearly seen in Fig. 3 . This correlation reflects the response of the ion density to $P_{\text {rf }}$.

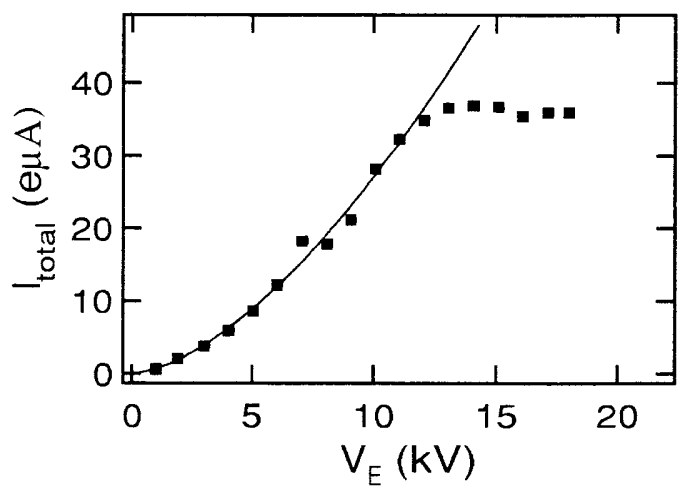

FIG. 1. $I_{\text {total }}$ as a function of $V_{E}$ measured at $P_{\mathrm{rf}}=13 \mathrm{~W}$. The solid line indicates the result analyzed by a power function. 


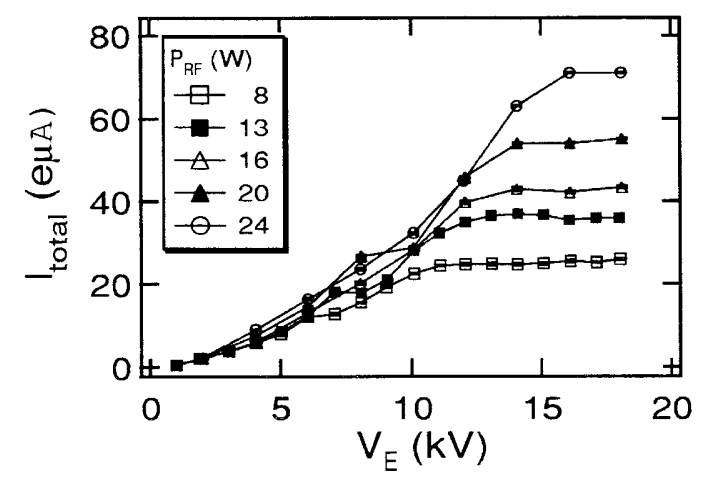

FIG. 2. $I_{\text {total }}$ as a function of $V_{E}$ measured at $P_{\mathrm{rf}}=12-80 \mathrm{~W}$.

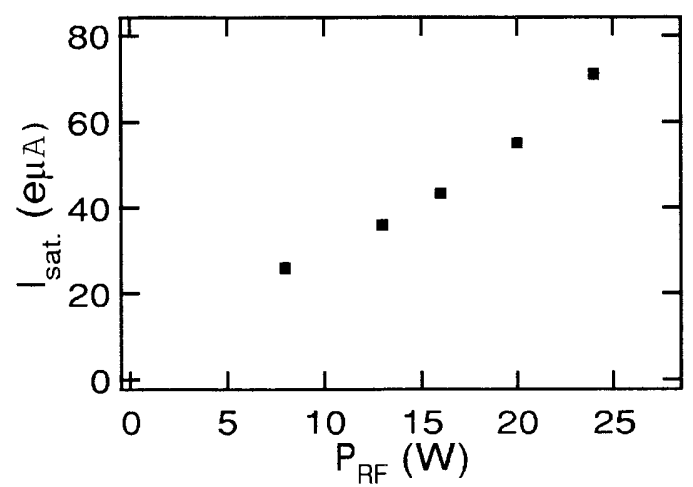

FIG. 3. $I_{\text {sat }}$ as a function of $P_{\mathrm{rf}}$.

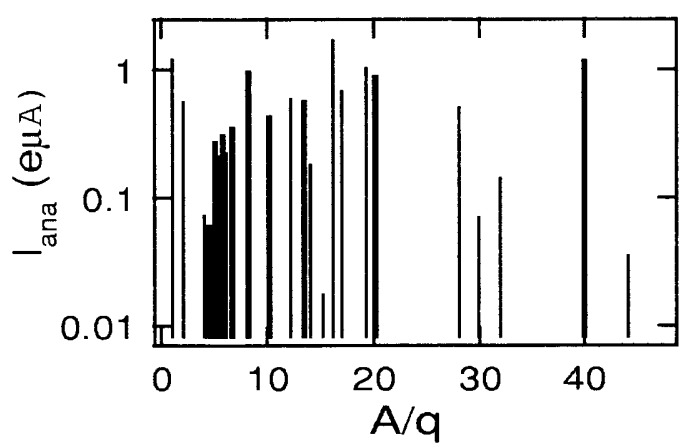

FIG. 4. Mass spectrum of ion beam measured at $P_{\mathrm{rf}}=22 \mathrm{~W}$. The thick lines and thin lines represent $\mathrm{Ar}$ ions and other ions, respectively.

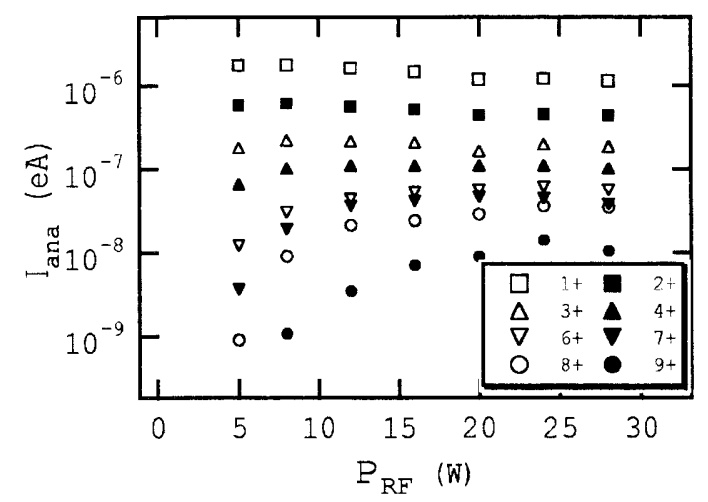

FIG. 5. Beam current of Ar ions as a function of $P_{\mathrm{rf}}$.

To check the ionization property of NANOGAN, the mass spectrum of the beam extracted from NANOGAN was observed by using a dipole magnet. Ar gas and air were fed into NANOGAN. $P_{\text {rf }}$ was varied over the range of 5-28 W and $V_{E}$ was $15 \mathrm{kV}$. The typical mass spectrum measured at $P_{\mathrm{rf}}=22 \mathrm{~W}$ is shown in Fig. 4. In the figure, we can find $\mathrm{Ar}$ ions, whose charges are $q=1-9$, indicated by thick lines. Furthermore, we can find other single-atom ions and molecular ions, which were originated from air, indicated by thin lines. It is concluded from these results that all of the atoms and molecules, which were fed into NANOGAN as a gas, could be ionized. In Fig. 5, the beam current $I_{\text {ana }}$ of Ar ion is shown as a function of $P_{\mathrm{rf}}$ for each charge state. In this figure, it is found that $\mathrm{I}_{\text {ana }}$ 's of $\mathrm{Ar}^{1+}$ and $\mathrm{Ar}^{2+}$ gradually decrease with $P_{\mathrm{rf}}$. Also, $I_{\text {ana }}$ 's of ions with $q>5$ increase with $P_{\text {rf }}$.

It is concluded from the above-mentioned measurements that the facility built at KUT has been working normally, except for the irradiation system. To study the formation of nanostructures with highly charged ions, further developments are required. A beam-profile monitor and an adequate irradiation system should be installed. To obtain a variety of probable ion beams for fabrication, optimization of the operation parameters of NANOGAN should be carried out with Ar and other elements.

${ }^{1}$ J. Orloff, Rev. Sci. Instrum. 64, 1105 (1993),

${ }^{2}$ D. M. Longo. W. E. Benson, T. Chraska, and R. Hull, Appl. Phys. Lett. 78. 981 (2001).

${ }^{3}$ R. E. Marrs, P. Beiersdorfer, and D. Schneider, Phys. Today 47, 27 (1994). ${ }^{4} \mathrm{C}$. Bieth et al., Proceedings of the 14th International Workshop on ECR Sources, Geneva, 1999, p. 147.

${ }^{5}$ http://www.pantechnik.net 